\title{
Effect of Oleic Acid Coating of Iron Oxide Nanoparticles on Properties of Magnetic Polyamide-6 Nanocomposite
}

\author{
RANJEETKUMAR GUPTA, ${ }^{1}$ KETAN PANCHOLI $10,1,3$ \\ RULSTON DE SA, ${ }^{1}$ DUNCAN MURRAY,${ }^{1}$ DEHONG HUO,${ }^{2}$ \\ GHAZI DROUBI, ${ }^{1}$ MAGGIE WHITE ${ }^{2}$ and JAMES NJUGUNA ${ }^{1}$
}

\begin{abstract}
1.-School of Engineering, Robert Gordon University, Sir Ian Wood Building, Garthdee Rd, Aberdeen AB10 7GE, UK. 2.-School of Engineering, Newcastle University, Newcastle upon Tyne NE1 7RU, UK. 3.—e-mail: k.pancholi2@rgu.ac.uk
\end{abstract}

This work reports the development and testing of a magnetic polymer (Polyamide 6, PA6) nanocomposite capable of melting when exposed to an external magnetic field. Addition of high concentrations of iron oxide nanoparticles (NPs) can induce quick melting but is detrimental to the mechanical properties of the polymer. To reduce the amount of NPs required for achieving efficient melting, they should be well dispersed in the polymer. In this study, the oleic acid loading on the surfaces of the NPs was varied to study the effect of variations in coatings on the dispersion in the polymer and on the polymer melting time. The NPs functionalized with oleic acid were added to melted monomer $\varepsilon$-caprolactam and polymerized using ring-opening polymerization. The resulting PA6 nanocomposite was characterized by Fourier-transform infrared spectroscopy, differential scanning calorimetry, x-ray diffraction and transmission electron microscopy. The results confirmed that the PA6 nanocomposite showed a decrease of 8-10\% in its glass-transition temperature compared to commercial PA6. The crystallinity of the synthesized samples were found to vary between $42 \%$ and $57 \%$. The $55 \mathrm{wt} . \%$ oleic acid-loaded NPs were found to disperse most efficiently in the PA6 matrix; however, some large agglomerates were formed due to excessive oleic acid. Therefore, the 22 wt.\% oleic acid coating showed overall superior dispersion. Additionally, the magnetic induction response was tested by observing a melt-characteristic of the magnetic polymer composite using a model set-up. Oleic acid concentration is found to affect the dispersion, melting time and crystallinity of the nanocomposite.

\section{INTRODUCTION}

Polymer nanocomposites (PNCs) that can respond to external stimuli, namely electric, optical or magnetic, are proving promising for various applications, such as drug delivery, information storage, electromagnetic interference shielding, magnetic resonance imaging, environmental remediation, microscale manufacturing and self-healing. ${ }^{1-5}$ Nanoparticles (NPs) naturally exhibit a strong tendency to agglomerate, as this minimizes the energy interactions linked with their high surface area to volume ratio. Moreover, increasing NP loading reduces the inter-particle distances, allowing the formation of the agglomerates due to short range van der Waals or hydrogen bonds, as observed in particle aggregation at the air-liquid interface in bubbles. $^{3,4}$ The presence of NP agglomerates in the polymer matrix causes an uneven material heating in response to the applied stimulus, as the region with agglomerated particles will quickly absorb much of the stimulus energy and will melt the surrounding polymer excessively or undergo pyrolysis. ${ }^{5}$ Therefore, mechanical and chemical dispersion techniques can be employed to achieve uniform heating of the polymer in nanocomposites.

To improve the NP dispersion efficacy, it is critical to adjust the level of interaction and bonding strength between the NPs and the surrounding polymer matrix. ${ }^{6}$ Various mechanical techniques 
such as melt compounding, ${ }^{7,8}$ high shear mixing, ${ }^{9}$ three roll mills, ${ }^{10}$ and solution blending ${ }^{11}$ are used to obtain uniform dispersion and to limit the formation of agglomerates. However, these methods do not promote a strong enough interaction between phases such that the agglomerates break down to a desired nanoscale. For example, the solution blending method still faces a major shortcoming due to the need to find an appropriate polymer-solvent pair for each filler-polymer system. ${ }^{12}$ Functionalization of NPs involving ionic charge, polymer coating (brushes), small molecules, and core-shell arrangement are efficient ways to promote their dispersion in the hydrophobic polymer matrix, when employed with electrostatic or steric mechanisms.

These techniques enhance the polymer-nanocomposite interactions at the nanoscale to confine agglomerate formation and hence many studies have analyzed the effect of NP functionalization on its adhesion with the base polymer matrix. ${ }^{13-16}$ The in situ polymerization method of dispersion has been successful for polyamide- and epoxy-based composites $^{17,18}$ as it enhances covalent bonding between the base polymer matrix and the NP surfaces. ${ }^{19}$ However, a high viscosity and nonNewtonian rheology of the melted polymer in the melt-blending method still poses a challenge in dispersing NPs homogenously. In in situ polymerization, ${ }^{20}$ the inorganic precursors are added in a low-viscosity melt-monomer to achieve optimum dispersion. Additionally, an in situ polymerization method has further opportunities for attaining rapid and controlled cross-linking density, and therefore it provides new properties to the PNC. The methods such as an interfacial polymerization, free radical polymerization, anionic ring-opening polymerization, frontal polymerization, micellar copolymerization, and network polymerization have already been studied for in situ polymerization. ${ }^{21}$

Considerable variations in the size to surface area ratios of NPs and their agglomerates affect the magnetic response nonlinearly, hence the functionalization of such NPs can help tune the anomalous behavior of the derived nanocomposites. ${ }^{22}$ The favoured NP morphology and crystalline phases obtained after functionalization can significantly affect the magnetic properties and crystallinity of the synthesized nanocomposites. ${ }^{23}$

The use of oleic acid as a capping agent results in hydrophobic coating, as polar end groups attach to the NP surface and form a strongly bonded protective monolayer, which enhances the uniform dispersion of the NPs. ${ }^{24}$ In order to study the effect of oleic acid (OA) loading in the coating of iron oxide NPs on nanocomposite properties, a magnetic nanocomposite was prepared by keeping the 1 wt.\% iron oxide NP concentration constant but varying the OA loading on the surface of the NPs. To prepare the nanocomposite, the 1 wt.\% NPs were blended in a low-viscosity melted monomer, $\varepsilon$-caprolactam (CL). Rapid polymerization was then achieved by a bulk anionic ring-opening polymerization. ${ }^{25}$ The use of a strong Grignard reagent ensured the rapid confinement of the NPs and the promotion of a uniform dispersion state. ${ }^{26} \mathrm{~A}$ total of three different bulk samples of the nanocomposite were prepared with 1 wt.\% iron oxide NPs, but the OA loading was kept at 22 wt. $\%, 55$ wt.\%, and 0 wt.\% (Table I). The prepared materials have promising applications in diverse scientific fields. ${ }^{27}$

\section{MATERIALS AND METHODS}

\section{Materials}

The following materials were used as received from Sigma-Aldrich, Dorset, UK: iron (III) oxide NPs (<50 nm size BET), oleic acid (OA) (99\% purity), $N$-acetylcaprolactam (NACL) (99\% purity), 3.0 M ethyl magnesium bromide (EtMgBr) solution in diethyl ether, and $\varepsilon$-caprolactam (CL) (99\% purity).

\section{Experimental Methods}

\section{Functionalization of Iron Oxide NPs}

The nanoparticle suspensions were prepared by dispersing $0.225 \mathrm{~g}$ of iron oxide NPs in $10 \mathrm{~mL}$ of methanol. Subsequently, OA was added into two suspension batches of $10 \mathrm{~mL}$ to make up $22 \mathrm{wt} . \%$ and $55 \mathrm{wt} . \%$ solutions. The mixture was rigorously stirred for $10 \mathrm{~min}$ at full power using a probe-type 150-W sonicator (Soniprep 150; MSE., UK). The NPs were thoroughly washed three times using methanol and decanted following centrifugation at $4000 \mathrm{rpm}$ for $5 \mathrm{~min}$. The NPs were then calcined at $80^{\circ} \mathrm{C}$ overnight in a furnace.

\section{In Situ Synthesis of Iron Oxide Polymer}

The first step in the polymerization process was to melt $30 \mathrm{~g}$ of $\mathrm{CL}$ at $60^{\circ} \mathrm{C}$. Iron oxide NPs were introduced into the melted monomer to prepare a 1 wt.\% iron oxide nanocomposite. The mixture of NPs and monomer was sonicated at $20 \mathrm{kHz}$ for $30 \mathrm{~min}$ to ensure effective dispersion of the NPs. The temperature of the mixture was then raised to $150^{\circ} \mathrm{C}$ and $0.86 \mathrm{~mL}$ of $\mathrm{EtMgBr}$ (at a concentration of $2.5 \mathrm{~mol} . \% \mathrm{~mol}^{-1} \mathrm{CL}$ ) was introduced under an inert gas atmosphere. To complete the polymerization, $0.94 \mathrm{~mL}(2.5 \mathrm{~mol} . \%)$ of NACL was added at $160^{\circ} \mathrm{C}$. The mixture instantly polymerized and captured the dispersed positions of the NPs. Before characterization, the nanocomposite was thoroughly washed with deionized water $(18 \mathrm{M} \Omega \mathrm{cm})$ at $100^{\circ} \mathrm{C}$ to remove any unreacted monomer, activator and initiator. The samples prepared for characterization are shown in Table I.

\section{Characterization Methodology}

The nanocomposite samples were characterized using a Perkin-Elmer ATR-FTIR (Attenuated Total Reflection-Fourier-Transform Infrared Spectroscope) 
Effect of Oleic Acid Coating of Iron Oxide Nanoparticles on Properties of Magnetic Polyamide6 Nanocomposite

Table I. Details of the prepared samples

\begin{tabular}{|c|c|c|c|}
\hline $\begin{array}{l}\text { Sample } \\
\text { description. }\end{array}$ & $\begin{array}{l}\text { OA loading on surface of iron oxide NPs } \\
(\text { wt.\%) }\end{array}$ & $\begin{array}{c}\text { Iron oxide NPs concentration } \\
(\text { wt.\%) }\end{array}$ & $\begin{array}{l}\text { Polymer } \\
\text { matrix }\end{array}$ \\
\hline No coating & 0 & 1 & PA6 \\
\hline 22 wt. $\%$ & 22 & 1 & PA6 \\
\hline 55 wt. $\%$ & 55 & 1 & PA6 \\
\hline Pristine polymer & 0 & 0 & PA6 \\
\hline
\end{tabular}

PA6 Magnetic polymer, Polyamide 6.

using a DGS-KBr sensor to identify functional groups and structural changes after the addition of the iron oxide NPs. Nanocomposite films averaging $0.1 \mathrm{~mm}$ thickness were prepared and a total of 30 scans in the range of $525-4000 \mathrm{~cm}^{-1}$ wavenumber were carried out at a resolution of $4 \mathrm{~cm}^{-1}$.

DSC was performed on a TA Instruments DSC $\mathrm{Q} 100$ at a heating rate of $10^{\circ} \mathrm{C} / \mathrm{min}$ under a nitrogen purge within the temperature range of $20-250^{\circ} \mathrm{C}$. The glass transition temperature $\left(T_{\mathrm{g}}\right)$ and melting temperature $\left(T_{\mathrm{m}}\right)$ were determined.

A PANalytical X'Pert Pro multipurpose diffractometer fitted with an X'Celerator detector and using monochromated $\mathrm{Cu}-\mathrm{K} \alpha$ radiation $(\lambda=1.54 \AA)$ was used to detect the crystalline phases present in the samples. X-ray diffraction (XRD) datasets were collected over a range of $10^{\circ}-65^{\circ} 2 \theta$ with a step size of $0.117^{\circ} 2 \theta$ and nominal time per step of $1099.82 \mathrm{~s}$. Phase identification was carried out using the HighScore Plus software (v.4.7) in conjunction with the ICDD Powder Diffraction File 4 Minerals (2017) and the ICDD Powder Diffraction File 2 (2004) databases.

To prepare samples for TEM study, an ultrathin section of the nanocomposite obtained using a microtome was placed on a 400-mesh-size gilder grid. A Philips CM100 TEM with an accelerating voltage of $100 \mathrm{kV}$ and a spot size of $10 \mathrm{~nm}$ was used to capture the images. The exposure time was varied from 0 to $50 \mathrm{~s}$, whereas the direct magnifications used ranged from $\times 7900$ to $\times 245,000$.

Small-angle x-ray scattering (SAXS) patterns were obtained using the polymer or nanocomposite films of $250-500 \mu \mathrm{m}$ thickness, on a Bruker AXS Nanostar equipped with a 2D Hi-STAR multi-wire gas detector and modified with a Xenocs GeniX 3D $\mathrm{x}$-ray source $(\mathrm{CuK} \alpha$ radiation with $\mathrm{x}$-ray wavelength $\lambda=1.54 \AA$ ) and motorized collimating scatterless slits. The patterns were obtained over a scattering vector length within the range of $0.008 \AA^{-1}<q<$ $0.18 \AA^{-1}$, where $q=\frac{4 \pi}{\lambda} \sin \theta$ and $\theta$ is a half of the scattering angle. One-dimensional scattering curves were obtained by an azimuthal binning and averaging of corresponding two-dimensional scattering patterns using software packages supplied with the SAXS instrument.

\section{RESULTS AND DISCUSSION}

In order to determine the effect of OA loading in the coating of the NPs on the PA6 properties during in situ polymerization, a PNC with 1 wt.\% iron oxide was prepared.

\section{Transmission Electron Microscopy}

The organic OA layer on the NPs was initially characterized by comparing TEM images of bare and coated NPs with different weight percentages of OA. A droplet of low-concentration aqueous NP suspension was placed on the TEM grid and dried before imaging. Care was taken to disperse the NPs well in the water using a sonicator. In addition, the degree of dispersion and/or agglomeration in the synthesized nanocomposite samples were studied using TEM images.

In order to measure the average diameter of NPs using TEM images (Fig. 1a, b and c, image analysis was performed (Supplementary material). The results show that the bare iron oxide NPs are highly agglomerated with sizes in the range of 35$50 \mathrm{~nm}$, while OA-coated NP diameters were found to be in range of $25-40 \mathrm{~nm}$ for $22 \mathrm{wt} \%$ and 30 $60 \mathrm{~nm}$ for $55 \mathrm{wt} \%$ OA loading. The hydrophobic nature of the organic OA layer on the NP surfaces restricted the interaction between the NPs and, hence, the mean diameter of the agglomerates in the polymer. ${ }^{28}$ The adsorption of OA on the NP surface is less transparent to the electron beam and, therefore, the coating on the NPs was observed as a thick opaque layer, as seen in Fig. 1a, b and c.

The TEM images of 50- $\mu \mathrm{m}$-thick microtome samples of the PNC were analyzed to investigate the extent of dispersion. As seen in Fig. $2 \mathrm{a}, \mathrm{b}$ and c, the dispersion was found to improve with increasing OA loading as the density of adsorbed hydrophobic molecules on the surface of the NPs increases. With $1-\mu \mathrm{m}$ and $60-\mathrm{nm}$ agglomerates were found in the PNC containing uncoated NPs and 55 wt.\% OAcoated NPs, respectively, albeit overloading of the OA coating led to the formation of many oversized agglomerates with overall smaller mean diameters. Hence, as observed from the TEM images, the NP agglomerates in the 55 wt.\% OA samples are larger in comparison to those observed in the $22 \mathrm{wt} . \%$ OA 

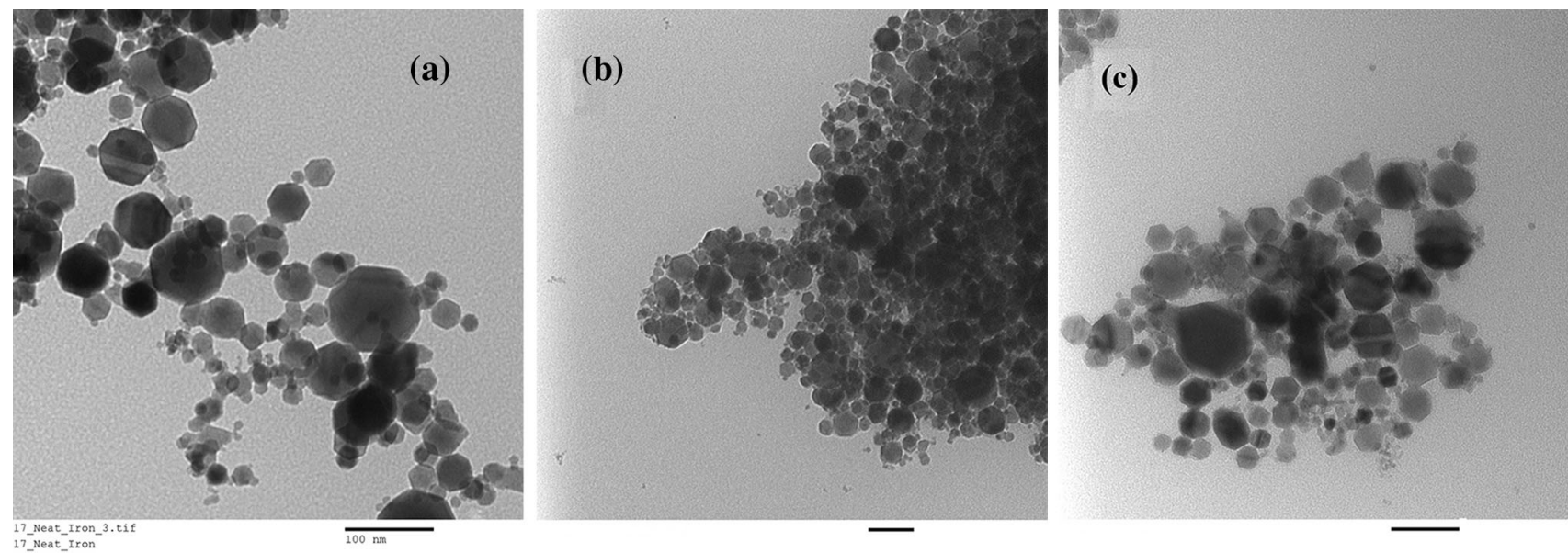

Fig. 1. (a) TEM image of uncoated iron oxide NPs (scale bar $100 \mathrm{~nm}$ ). TEM images of OA-coated NPs: (b) $22 \%$ OA and (c) $55 \%$ OA (scale bar $100 \mathrm{~nm})$.
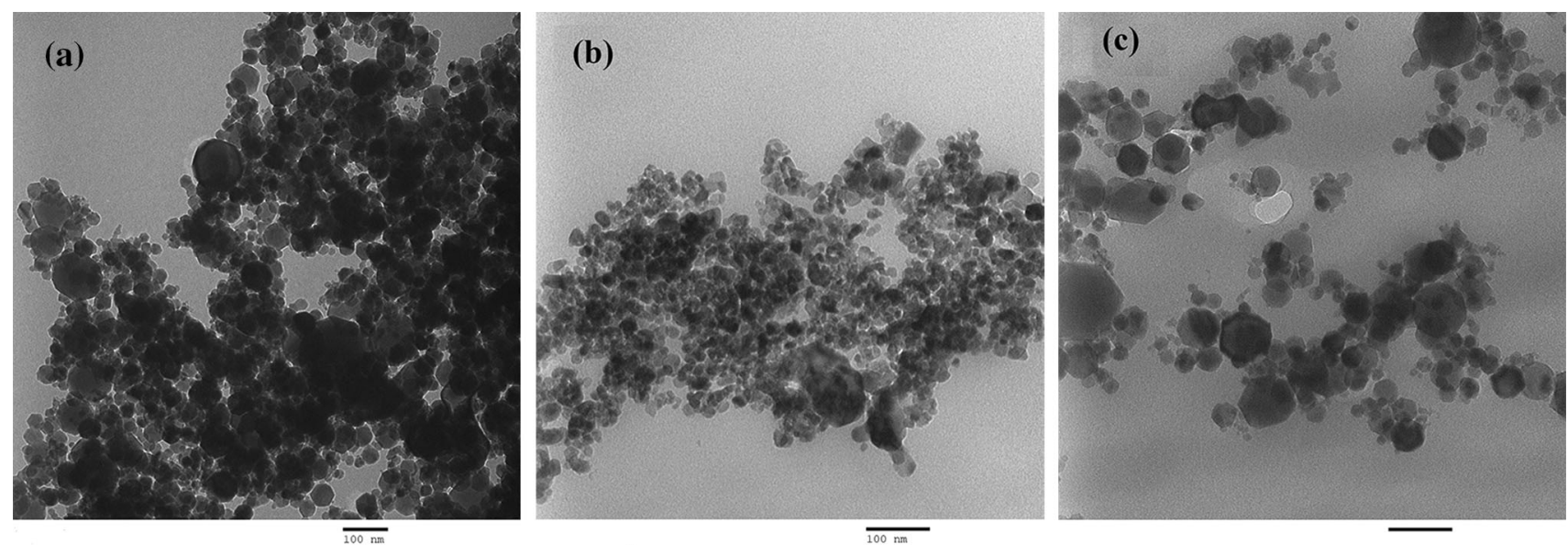

Fig. 2. TEM images show dispersion of NPs in (a) PNC with uncoated NPs, (b) PNC with NPs coated with 22 wt.\% OA loading, and (c) PNC with NPs coated with 55 wt.\% OA loading (scale bar $100 \mathrm{~nm}$ ).

samples. This is comparable with the size calculated from the XRD peaks and the SAXS results.

\section{Fourier-Transform Infrared Spectroscopy}

The FTIR peaks observed have been found to match with all the standard template chemical group peaks of the commercial PA6, which confirmed its successful synthesis. As seen in Fig. 3, a peak attributed to hydrogen bonds and $\mathrm{N}-\mathrm{H}$ stretching was observed at $3293 \mathrm{~cm}^{-1}$. Further, the $\mathrm{CH}_{2}$ asymmetric and symmetric stretch of PA6 can be assigned to the peaks observed at $2934 \mathrm{~cm}^{-1}$ and $2861 \mathrm{~cm}^{-1},{ }^{29,30}$ respectively.

Also, the shift in the spectra observed for the OAcoated samples, i.e. the new shifted peaks observed at $2929 \mathrm{~cm}^{-1}(22 \mathrm{wt} . \% \mathrm{OA})$ and $2921 \mathrm{~cm}^{-1}(55 \mathrm{wt} . \%$ $\mathrm{OA})$ and the peaks of $2860 \mathrm{~cm}^{-1}$ (22 wt.\% OA) and $2862 \mathrm{~cm}^{-1}$ (55 wt.\% OA) are attributed to the asymmetric and symmetric $\mathrm{CH}_{2}$ stretching, respectively. These correspond to the characteristic OA absorption peaks of $2924 \mathrm{~cm}^{-1}$ and $2854 \mathrm{~cm}^{-1}$, respectively. ${ }^{31,32}$ Even the slight peaks developed at $1716 \mathrm{~cm}^{-1}$ (22 wt.\% OA) and $1715 \mathrm{~cm}^{-1}$ (55 wt.\% OA) are due to the overlapping of carboxyl groups and the OA double-bond absorption bands. This confirms the presence of chemisorbed OA (compared to the standard $1710 \mathrm{~cm}^{-1}$ peak) that acts as a surfactant, ${ }^{32}$ improving the dispersion (which has been studied from the TEM, XRD and SAXS results). Also, the inset image shows that a slight OA peak developed, matching with the standard peak at $1710 .^{32}$

The shift in the peaks from $1536 \mathrm{~cm}^{-1}$ to $1540 \mathrm{~cm}^{-1}$ can be attributed to a decrease in the degree of crystallinity. ${ }^{33}$ Additionally, this band at $1536-1635 \mathrm{~cm}^{-1}$ also corresponds to the primary amide band of amide II-type. The peaks around $1536 \mathrm{~cm}^{-1}$ and $1540 \mathrm{~cm}^{-1}$ correspond to the mentioned amide II of the primary type, resulting in vibration due to the bending of CO-NH (939$948 \mathrm{~cm}^{-1}$ ) and the N-H bond. ${ }^{34}$ The intensity and position of these crystalline bands determines the 
Effect of Oleic Acid Coating of Iron Oxide Nanoparticles on Properties of Magnetic Polyamide6 Nanocomposite

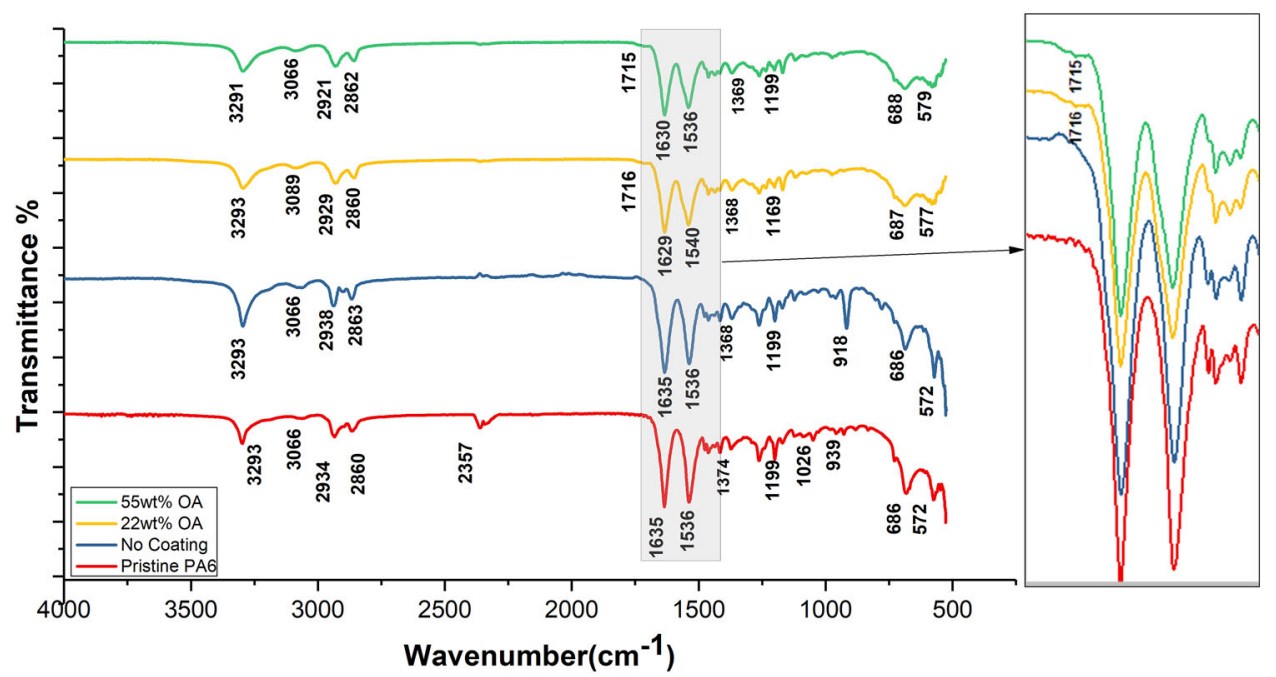

Fig. 3. ATR-FTIR spectra of pristine PA6, PNC containing uncoated NPs and PNC containing NPs with varying proportions of OA coatings.

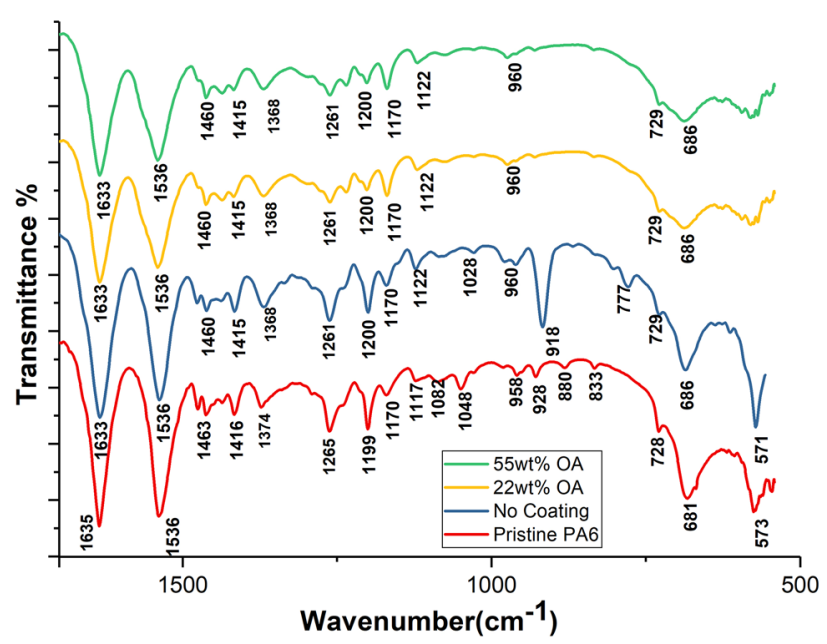

Fig. 4. ATR-FTIR spectra of polymer samples with pristine PA6, no coating PNC and PNC with varying proportions of OA coatings.

degree of crystallinity, whereas the broader peaks of these bands suggest the formation of an amorphous phase. $^{35}$ Therefore, it can be inferred that the polymer with 22 wt.\% OA has a moderately higher degree of crystallinity, as the amide II band was sensitive to the crystalline structure.

Hence, the increasing concentration of $\mathrm{OA}$ employed lowers the crystallinity. Furthermore, the polymer chain mobility is limited and the crystallization process slackened ${ }^{36}$ by the carboxylic end group at the end of the fatty acid chain in the OA coating interacting with $\mathrm{NH}_{2}$ of the polyamide chain. The wavelength fingerprint for $\mathrm{OA}$ at around $1629 \mathrm{~cm}^{-1}$ corresponding to the asymmetric and symmetric bands (COO-) of the oleic acid grafted at the surface of the iron oxide NPs were suppressed by PA6 and observed as flat peaks, as seen in Fig. $4 .{ }^{37,38}$ The peak near $686 \mathrm{~cm}^{-1}$ corresponds to both the Fe-O bond stretching of iron oxide and the amide $V(\alpha$ and $\beta)$ of the pristine PA6. ${ }^{39}$ However, interaction between the peaks results in the suppression of the peak near $686 \mathrm{~cm}^{-1}$ for the $22 \mathrm{wt} . \%$ and 55 wt.\% PNCs. The increased interaction between the NPs and the polymer matrix due to the increase in OA loading is further confirmed with DSC results.

All the nanocomposites contained 1 wt.\% iron oxide NPs, and the $\mathrm{Fe}-\mathrm{O}$ peaks are not clearly discernible in the FTIR plots. To clearly establish the effect of NP inclusions on PA6, the FTIR spectrum of PNC containing a high weight percentage, i.e., 20 wt.\% of NPs, was obtained and compared with the pristine PA6 and the PNC containing uncoated iron oxide NPs ("No coating" legend in Figs. 3 and 4, and Figure S1) spectra (which had only 1 wt.\% of NP inclusion). The spectra of 20 wt.\% PNC, as seen in Figure S1, shows a distinctive broadening of the peaks around the absorption bands of $295-541 \mathrm{~cm}^{-1}$, a characteristic of Fe-O stretching, ${ }^{40}$ and also the peak at $587 \mathrm{~cm}^{-1}$ confirms the inclusion of $\mathrm{Fe}_{3} \mathrm{O}_{4}$, ${ }^{41}$ especially from the 20 wt.\% sample. The distinguishing peak at $534 \mathrm{~cm}^{-1}$, a characterizing peak confirming the presence of iron oxide, is only observed in the 20 wt. $\%$ sample, whereas the $570 \mathrm{~cm}^{-1}$ is attributed to amide-V stretching. ${ }^{41}$ This implies that the $\mathrm{Fe}_{3} \mathrm{O}_{4}$ peak is not discernible due to the low wt.\% of iron oxide NPs samples, as seen in Figs. 3 and 4 . To confirm, energy dispersive X-ray spectroscopy (EDX) results were obtained (Supplementary Material Figure S2 a-d).

\section{Differential Scanning Calorimetry and Degree of Crystallinity}

Noticeable melting with endothermic peaks clearly depicts the glass transition $\left(T_{\mathrm{g}}\right)$ and the melting temperatures $\left(T_{\mathrm{m}}\right)$ of the polymer samples, as shown in Fig. 5 . The $T_{\mathrm{g}}$ occurrence of the pristine polymer is observed at around $46^{\circ} \mathrm{C}$, matching the previously reported values. ${ }^{42,43}$ 
However, the observed $T_{\mathrm{m}}$ values are slightly lower than previously reported values, at around $220^{\circ} \mathrm{C}{ }^{42,43}$ Additionally, as seen in Table II, the $T_{\mathrm{m}}$ of the nanocomposite was found to increase with increased OA loading. However, the uncoated NP sample showed subsequent reductions in the $T_{\mathrm{m}}$ and $T_{\mathrm{g}}$ of the sample due to the uncoated inclusions leading to amorphous growth and hence reduced crystallinity, as summarized in Table II.

Increased wetting of the surface-modified NPs by polymers is known to either increase or decrease the crystallinity and, hence, the $T_{\mathrm{g}}$ and $T_{\mathrm{m}}$ of the PNC. To confirm whether or not this is the case for the samples studied here, the percentage degree of crystallinity was calculated by dividing the total heat of fusion for each sample by the heat of fusion for $100 \%$ crystalline PA6, which is $190 \mathrm{~J} / \mathrm{g}$. ${ }^{44}$ The values for the degree of crystallinity, calculated from the areas under the curve for all the samples, and the glass transition $\left(T_{\mathrm{g}}\right)$ and melting temperature $\left(T_{\mathrm{m}}\right)$, are as listed in Table II.

The increase in $T_{\mathrm{g}}$ due to reductions in cooperative segmental mobility in the polymer is because the OA's denser fatty acid chains on the surface of the NPs induced greater bonding with the polymer matrix, as observed with the DSC trend. ${ }^{45-47}$ The strong attraction at the interface of the NPs and the polymer tends to decrease the crystallinity and $T_{\mathrm{m}}$, as observed in the DSC results (Table II).

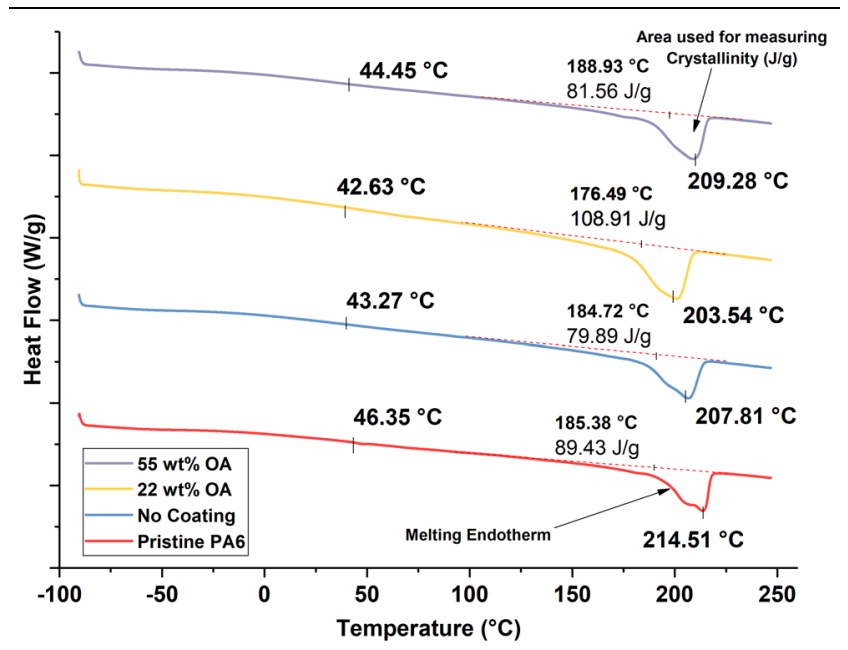

Fig. 5. DSC curves of the PA6 samples with varying percentages of $\mathrm{OA}$ coating.

\section{X-ray Diffraction}

The XRD results confirmed the polymer as the semi-crystalline phase. ${ }^{48}$ Diffraction peaks observed at $30.2^{\circ} 2 \theta(220), 35.6^{\circ} 2 \theta(311), 43.3^{\circ} 2 \theta(400)$, $57.25^{\circ} 2 \theta(511)$ and $62.9^{\circ} 2 \theta$ (440) (Fig. 6) were found to compare well with the JCPDS card number 01-089-5892 for the magnetic mineral $\gamma$-iron oxide (cubic maghemite), therefore confirming the spinel structure of the NP iron oxides. The crystal planes within the maghemite represented by the most intense diffraction peaks, described by the Miller indices above, were used in Eq. 1 to calculate the crystallite sizes of the NPs; Eq. 1 relates the diffraction peak width $(B)$ to the crystallite size $(L)$.

$$
B(2 \theta)=\frac{K \lambda}{L \cos \theta}
$$

As seen in Fig. 6, with 22 wt.\% OA loading, the prominent diffraction peaks showed a low intensity and maximum broadening, which suggests a decrease in NP agglomeration size. Similar to the TEM results, the standard deviation of the crystallite size for 55 wt.\% OA loading is high, confirming the formation of few large agglomerates. However, the mean size of the particles was found to be lower than $32.41 \mathrm{~nm}$, again with the large standard deviation (Table III).

Ideally, PA6 contains two monoclinic crystalline phases: the $\alpha$-phase and the $\gamma$-phase. With the entirety of the $\alpha$-phase attaining a trans-chain conformity, a hydrogen bond appears between adjacent antiparallel chains in this phase; the thermodynamically most stable phase. ${ }^{48}$ Conversely, the chains appear twisted in the $\gamma$-phase, enabling the formation of hydrogen bonds between parallel chains. ${ }^{48}$

In the PA6 samples prepared during this study, two broad-based diffraction peaks were observed around $21^{\circ} 2 \theta(4.2 \AA)$ and $24^{\circ} 2 \theta(3.7 \AA)$ which almost certainly correspond to the $\alpha$-phase of PA6. Figure 8 shows that the $\alpha$-phase dominates the crystalline structure of the PA6. The peak at $21^{\circ} 2 \theta$ (4.2 $\AA$ ) is referred to as $\alpha_{1}$ and originated from the (200) plane, whereas the peak at $24^{\circ} 2 \theta(3.7 \AA)$ originated from the $(002)$ plane. ${ }^{49}$ The minor peaks observed at $17^{\circ} 2 \theta, 22^{\circ} 2 \theta$ and $28^{\circ} 2 \theta$ can be identified as the $\gamma$-phase of of the PA6 with the corresponding Miller indices of (020), (001) and $(200) /(201){ }^{47}$

Table II. The degree of crystallinity, glass transition and melting temperature from DSC results

\begin{tabular}{|c|c|c|c|c|}
\hline Sample & $\boldsymbol{T}_{\mathrm{g}}\left({ }^{\circ} \mathbf{C}\right)$ & $\boldsymbol{T}_{\mathbf{m}}\left({ }^{\circ} \mathbf{C}\right)$ & Enthalpy $(J / g)$ & Degree of crystallinity (\%) \\
\hline Pristine PA6 & $46.35 \pm 1$ & $214.15 \pm 2$ & $89.43 \pm 2$ & $47.07 \pm 2$ \\
\hline No coating & $43.27 \pm 3$ & $207.81 \pm 3$ & $79.89 \pm 4$ & $42.05 \pm 4$ \\
\hline 22 wt.\% OA & $42.63 \pm 2$ & $203.54 \pm 3$ & $108.91 \pm 4$ & $57.32 \pm 4$ \\
\hline 55 wt.\% OA & $44.45 \pm 1$ & $209.28 \pm 2$ & $81.56 \pm 2$ & $42.92 \pm 2$ \\
\hline
\end{tabular}


Effect of Oleic Acid Coating of Iron Oxide Nanoparticles on Properties of Magnetic Polyamide6 Nanocomposite

There are various factors contributing to the observed diffraction patterns (Fig. 6). The two prominent peaks related to the $\alpha$ crystalline phase of PA6 were observed to decrease with increased OA loading up to $22 \mathrm{wt} . \%$, suggesting an increase in the crystalline to amorphous ratio, meaning the degree of crystallinity. The XRD plots presented here suggest that the maghemite iron oxide NPs tend to be more crystalline and ordered than the polymer PA6, which is reported as being semi-crystalline ${ }^{48}$ and therefore less well ordered.

Both the TEM and XRD results suggest that the 22 wt.\% OA proportion of NP coating, the crystallite size, and therefore the NP agglomerate size, are the lowest. In summary, small crystal formation results due to improved dispersion of NPs. ${ }^{50,51}$

\section{Small Angle X-ray Scattering}

The filler dispersivity in a PNC has been studied using scattering techniques. Statistical mechanical theories relate to the dispersion state of the nanoinclusions as well as the stress carried by the polymer chains. ${ }^{52}$ The model-free approach of average particle size determination using the SAXS data is based on the implementation of the Guinier and Porod law. ${ }^{53,54}$

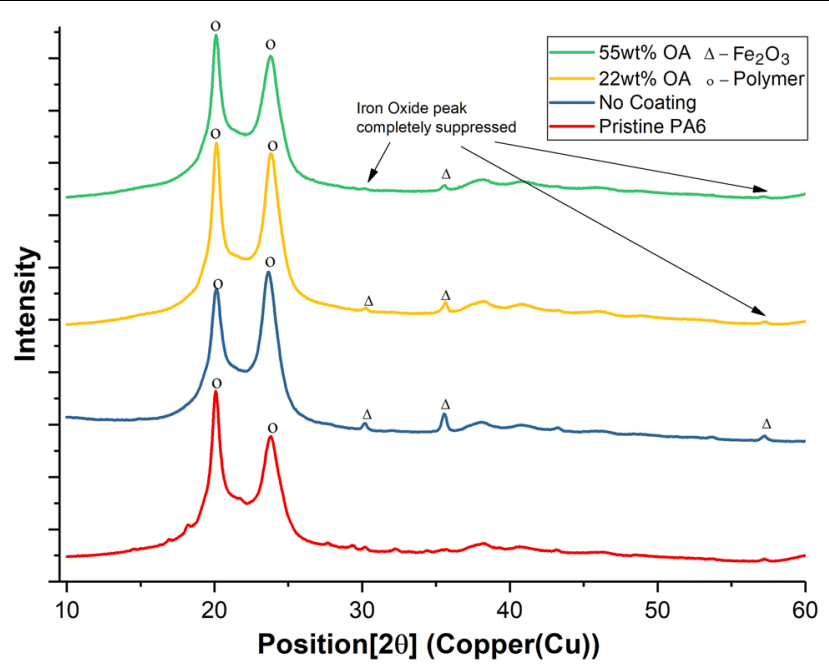

Fig. 6. XRD patterns of the PA6 samples with varying percentages of OA loading of coatings on the NPs. The triangle $(\Delta)$ and circle $(\bigcirc)$ represents the peaks related to iron oxide and polymer, respectively.
Figure S3 represents the scattering intensity $I(q)$, plotted as a function of the scattering vector $q$. The SAXS profile shows two regions: the flat response from PA6 and the high gradient response from the nanoparticles in the samples. The cumulatively slope-dropping region is a characteristic of a Porod scattering response from the NPs. ${ }^{55}$ The Guinier region precedes the Porod region, which reflects the radius of gyration of the NPs according to the Guinier's law:

$$
I(q)=G \exp \left(-\frac{q^{2} R_{\mathrm{g}}^{2}}{3}\right)
$$

where $G$ is the Guinier prefactor and $R_{\mathrm{g}}$ is the radius of gyration. The slope of the regions in the Guinier plot represented in Fig. 7 are used to determine $R_{\mathrm{g}}$.

Using Eq. 3, the mean diameter $D$ is calculated: ${ }^{56,}$

$$
D=2 \times(5 / 3)^{1 / 2} R_{g}
$$

The results in Table IV are in proximity of those obtained with TEM and XRD above. This confirms that the 22 wt.\% OA sample has the lowest NP size and also has the fewest agglomerates. The maximum standard deviation was found to be $\pm 8.9 \mathrm{~nm}$ for the uncoated particles.

\section{Melt Characteristics}

A magnetic field response test of the synthesized nanocomposite was carried out to determine its melt characteristics. The magnetic coil (30 V) set-up used (Figure S4) was designed ${ }^{58}$ and used for testing.

Preliminary melting times upon exposure to the magnetic field were recorded, as shown in Table V. A total of five sets of results for each nanocomposite film $(200 \mu \mathrm{m}$ thick), with $22 \%$ and 55 wt. $\%$ OA loading, were recorded. A representative surfaces of nanocomposite samples with defects are shown in Fig. $8 \mathrm{a}$ and c. The same defective nanocomposites were exposed to a magnetic field to observe the surface melting and therefore healing effect. Typical results showing surface crack healing in samples containing iron oxide NPs coated with $22 \%$ and 55 wt.\% OA loading are shown in Fig. 8b and d.

Both samples confirmed the melting response as reported earlier. ${ }^{58}$ The cracks identified on the initial samples were observed to heal after the

\begin{tabular}{|c|c|c|c|c|}
\hline \multirow[b]{2}{*}{ Sample } & \multirow[b]{2}{*}{ Peak position $(2 \theta)$} & \multirow[b]{2}{*}{ FWHM } & \multicolumn{2}{|c|}{ Absolute crystallite size } \\
\hline & & & Size $\left({ }^{\circ} \mathbf{A}\right)$ & Size (nm) \\
\hline No coating & $35.58 \pm 2$ & $0.2092 \pm 3$ & $417.57 \pm 2$ & $41.76 \pm 2$ \\
\hline 22 wt. $\%$ OA & $35.68 \pm 3$ & $0.2937 \pm 4$ & $300.17 \pm 3$ & $30.02 \pm 3$ \\
\hline 55 wt. $\%$ OA & $35.55 \pm 3$ & $0.2785 \pm 13$ & $324.15 \pm 13$ & $32.41 \pm 13$ \\
\hline
\end{tabular}

Table III. Crystallite size calculation from XRD results 


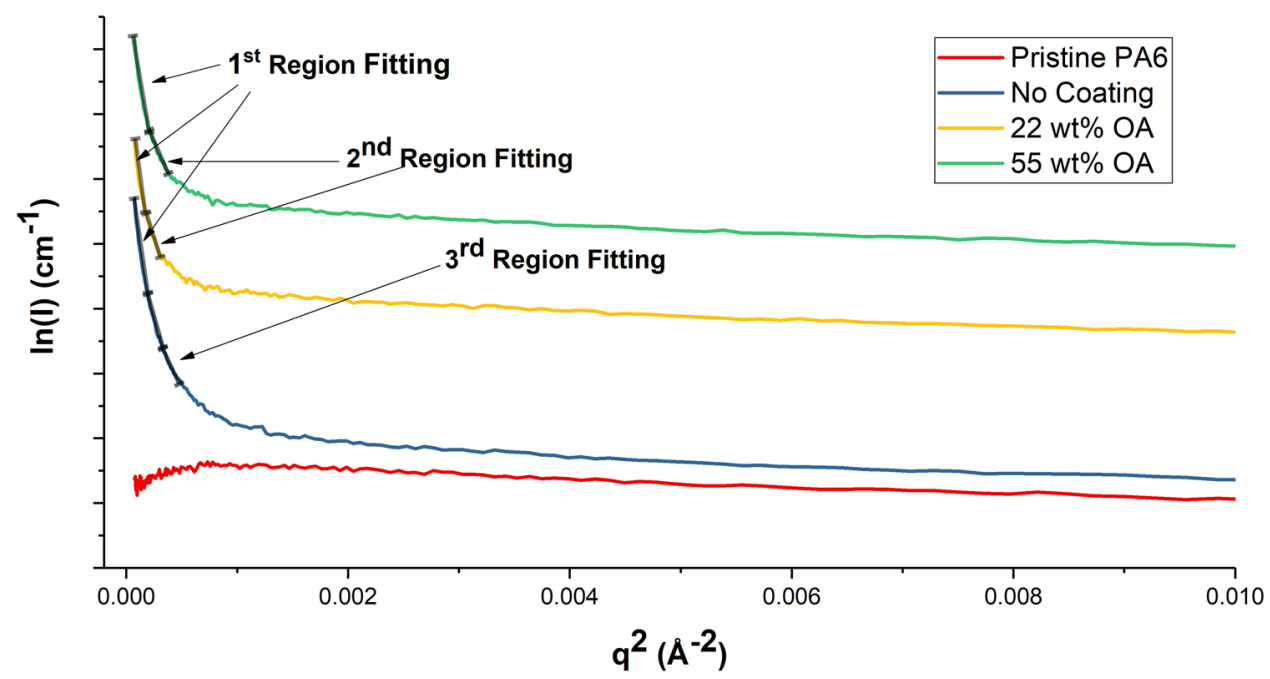

Fig. 7. Guinier plot for all the samples, with the region fitting highlighted.

Table IV. Mean NP size calculated from SAXS results

\begin{tabular}{lccc}
\hline Sample & No coating & 22 wt.\% OA & 55 wt.\% OA \\
\hline 1st region fitting & & & $0.009-0.012$ \\
$I(q)$ Range & $0.009-0.012$ & $0.009-0.014$ & 185.136 \\
$R_{\mathrm{g}}$ & 196.719 & 151.196 & 47.80 \\
$D(\mathrm{~nm})$ & 50.79 & 39.04 & $0.012-0.019$ \\
2nd region fitting & & & 121.676 \\
$I(q)$ Range & $0.012-0.018$ & $0.014-0.021$ & 31.41 \\
$R_{\mathrm{g}}$ & 143.985 & 94.057 & - \\
$D(\mathrm{~nm})$ & 37.17 & 24.29 & - \\
3rd region fitting & & & - \\
$I(q)$ Range & $0.018-0.022$ & - & - \\
$R_{\mathrm{g}}(\mathrm{nm})$ & 97.123 & - & \\
\hline
\end{tabular}

Table V. Magnetic induction melt-characteristic response of nanocomposite samples

\begin{tabular}{lc}
\hline Sample & Melt response time (s) \\
\cline { 3 - 3 } No particles & $\mathrm{NA}$ \\
Iron oxide, 22 wt.\% OA & $90 \pm 50$ \\
Iron oxide, 55 wt.\% OA & $90 \pm 15$ \\
\hline
\end{tabular}

magnetic induction heating response. These are marked in Fig. 8b and d showing - the feasibility of material healing on damaged samples. The mean time periods for the melt response of-five sample surfaces were found to be similar in both cases. This is not surprising given the NP wt.\% loading for both samples was the same at $1 \mathrm{wt} . \%$. However, the variance in the time recorded was found to be higher in-samples with the 22 wt.\% OA coating showing uncertainties.

The considerable variation in the melt response time for the 22 wt.\% OA sample can be attributed to the dispersion of the NPs. The agglomerates in the
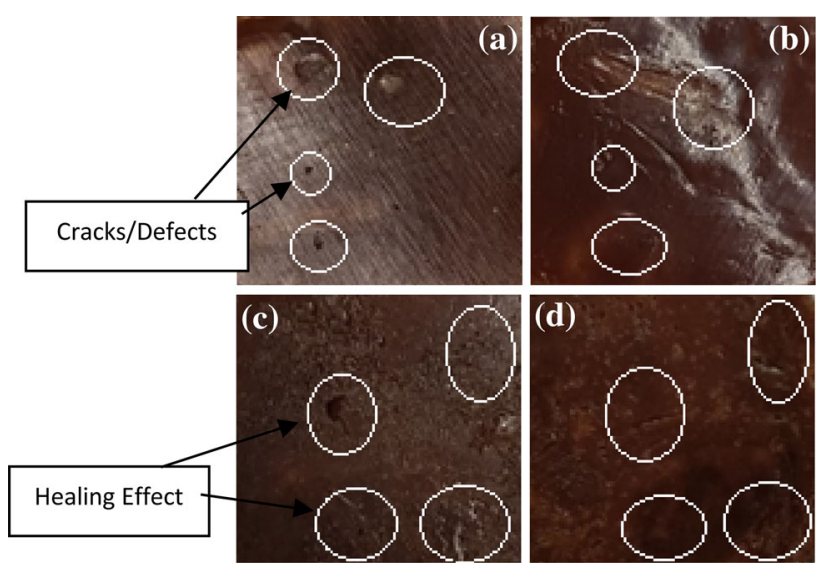

Fig. 8. Defective polymer nanocomposite films with (a) 22 wt.\% and (c) 55 wt. \% OA-loaded NPs. The same films after surface melting using magnetic induction with (b) 22 wt.\% and (d) 55 wt.\% OAloaded NPs.

55 wt.\% OA sample created localized heating effects. Hence, some samples melted quickly in certain regions. 
Effect of Oleic Acid Coating of Iron Oxide Nanoparticles on Properties of Magnetic Polyamide6 Nanocomposite

\section{CONCLUSION}

An iron oxide nanocomposite in a PA6 polymer matrix was successfully synthesized using an in situ polymerization approach. Increases in the OA concentration of the NP coating, up to $22 \mathrm{wt} \%$, showed improvement in the dispersion combined with increased $T_{\mathrm{g}}$, reduced $T_{\mathrm{m}}$ and decreased crystallinity, compared with a commercial PA6 polymer. The results confirmed changes in the properties of the polymer due to variation in OA loading in the coating of iron oxide NPs. In general, increases in OA concentration improved the dispersion of the iron oxide NPs and their chemical interaction with the host polymer chain. At 55 wt.\%, OA loading led to the formation of some agglomerates, and, although the mean diameter was indicating improved dispersion, the standard deviation was large. Furthermore, the overall result showed that the 22 wt.\% loading was optimum for improved dispersion of NPs. The magnetic response suggests that a higher weight percentage of NPs would improve the melting response time. Such nanocomposites can find use in biomedical applications. The effect of particle concentration and other polymerization catalysts will be researched in future work.

\section{ACKNOWLEDGEMENTS}

Ranjeetkumar thanks Robert Gordon University for Ph.D. financial support.

\section{CONFLICT OF INTEREST}

The author declares that they have no conflict of interest.

\section{OPEN ACCESS}

This article is distributed under the terms of the Creative Commons Attribution 4.0 International License (http://creativecommons.org/licenses/by/4.0/), which permits unrestricted use, distribution, and reproduction in any medium, provided you give appropriate credit to the original author(s) and the source, provide a link to the Creative Commons license, and indicate if changes were made.

\section{ELECTRONIC SUPPLEMENTARY MATERIAL}

The online version of this article (https://doi.org/ 10.1007/s11837-019-03622-5) contains supplementary material, which is available to authorized users.

\section{REFERENCES}

1. A.S. Perera, S. Zhang, S. Homer-Vanniasinkam, M. Coppens, and M. Edirisinghe, ACS Appl. Mater. Interfaces 10 , 15524 (2018).

2. C. Cih, K. Kun-Cheng, and Y. Sen-Yeu, RSC Adv. 7, 6336 (2017).

3. J. Fiabane, P. Prentice, and K. Pancholi, BioMed Res. Int. (2016). https://doi.org/10.1155/2016/3572827.

4. K. Pancholi, P.K.J. Robertson, P. Okpozo, N.S. Beattie, and D. Huo, J. Phys. Energy 1, 015007 (2018).

5. K. Pancholi, R. Gupta, R. Prabhu, D. Huo, M. Pancholi, V. Jha, J. Latto, and N. Dodds, OCEANS'17 MTS/IEEE, (Aberdeen, 2017).

6. R. Gupta, R. Prabhu, D. Huo, M. Pancholi, J. Njuguna, and K. Pancholi, 3rd Defence and Security Doctoral Symposium (DSDS17), (Cranfield University, 2017).

7. M. Zhang and R.P. Singh, Mat. Lett. 58, 408 (2004).

8. V. Yong and H.T. Hahn, Nanotechnology 15, 1338 (2004).

9. S. Lao, W. Ho, K. Ngyuen, J. Cheng, J.H. Koo, L. Pilato, and G. Wissler., SAMPE Fall Technical Conference-37th ISTC (2005).

10. J.H. Koo, L.A. Pilato, and G.E. Wissler, J. Spacecr. Rockets 44, 1250 (2007).

11. C. Chen, The Manufacture of Polymer Nanocomposite Materials Using Supercritical Carbon Dioxide (Doctoral dissertation, Virginia Tech., 2011).

12. Y. Chen, A.M. Kushner, G.A. Williams, and Z. Guan, Nat. Chem. 4, 467 (2012).

13. J. Tang, Y. Wang, H. Liu, Y. Xia, and B. Schneider, J. Appl. Polym. Sci. 90, 1053 (2003).

14. D. Ma, T.A. Hugener, R.W. Siegel, A. Christerson, E. Mårtensson, C. Önneby, and L.S. Schadler, Nanotechnology 16 , 724 (2005)

15. Y. Chen, S. Zhou, H. Yang, and L. Wu, J. Appl. Polym. Sci. 95, 1032 (2005).

16. A. Usuki, M. Kawasumi, Y. Kojima, A. Okada, and T. Kurauchi, Jpn. J. Polym. Sci. Technol. 52, 440 (1995).

17. Z. Spitalsky, G. Tsoukleri, D. Tasis, C. Krontiras, S.N. Georga, and C. Galiotis, Nanotechnology 20, 405702 (2009).

18. M. Liu, T. Zhu, Z. Li, and Z. Liu, J. Phys. Chem. C 113, 9670-9675 (2009).

19. J.H. Koo, L.A. Pilato, G. Wissler, A. Lee, A. Abusafieh, and J. Weispfenning, International SAMPE Symposium and Exhibition (Proceedings), Society for the Advancement of Material and Process Engineering, vol. 50, p. 1935 (2005).

20. Y. Chen, S. Zhou, H. Yang, and L. Wu, J. Appl. Polym. Sci. 95, 1032 (2005).

21. S. Laurent, D. Forge, M. Port, A. Roch, C. Robic, L. Vander Elst, and R.N. Muller, Chem. Rev. 108, 2064 (2008).

22. L.M. Bronstein, X. Huang, J. Retrum, M. Pink, B.D. Stein, and B. Dragnea, Chem. Mater. 19, 3624 (2007).

23. C. Bakkali-Hassani, D. Tunc, K. Roos, M. Planes, P. Lecomte, and S. Carlotti, Macromolecules 50, 175 (2016).

24. S. Wei, Q. Wang, J. Zhu, L. Sun, H. Lin, and Z. Guo, Nanoscale 3, 4474 (2011)

25. C. Wang, C. Xu, H. Zeng, and S. Sun, Adv. Mater. 21, 3045 (2009)

26. K.A. Al-Saad, M.A. Amr, D.T. Hadi, R.S. Arar, M.M. ALSulaiti, T.A. Abdulmalik, N.M. Alsahamary, and J.C. Kwak, Arab J. Nucl. Sci. Appl. 45, 335 (2012).

27. P.B. Shete, R.M. Patil, B.M. Tiwale, and S.H. Pawar, J. Magn. Magn. Mater. 377, 406 (2015).

28. G.K. Xu, W. Lu, X.Q. Feng, and S.W. Yu, Soft Matter 7, 4828 (2011).

29. N. Ngwuluka, Aaps Pharmscitech 11, 1603 (2010).

30. J. Pockett, Crystallinity in Linear Polyamides: A Study Using Melt Blending with Small-Molecule Diluents (Doctoral dissertation, University of South Australia, 2004). 
31. Y.F. Shen, J. Tang, Z.H. Nie, Y.D. Wang, Y. Ren, and L. Zuo, Sep. Purif. Technol. 68, 312 (2009).

32. M. Mahdavi, M. Ahmad, M. Haron, F. Namvar, B. Nadi, and J. Amin, Molecules 18, 7533 (2013).

33. M.K. Verhoefen, G. Schäfer, S. Shastri, I. Weber, C. Glaubitz, W. Mäntele, and J. Wachtveitl, Biochim. Biophys. Acta Bioenerg. 1807, 1583 (2011).

34. F. Yang, M. Zhao, B. Zheng, D. Xiao, L. Wu, and Y. Guo, J. Mater. Chem. 22, 25471 (2012).

35. C. Harrats and G. Groeninckx, Macromol. Rapid Commun. 29, 14 (2008).

36. M. Zhang, B.H. Guo, and J. Xu, Crystals 7, 4 (2016).

37. D. Tunc, H. Bouchekif, B. Améduri, C. Jérôme, P. Desbois, P. Lecomte, and S. Carlotti, Eur. Polym. J. 71, 575 (2015).

38. X.Y. Wang, D. Mertz, C. Blanco-Andujar, A. Bora, M. Ménard, F. Meyer, C. Giraudeau, and S. Bégin-Colin, RSC Adv. 6, 93784 (2016).

39. S.T. Navale, G.D. Khuspe, M.A. Chougule, and V.B. Patil, RSC Adv. 4, 27998 (2014).

40. R. Khandanlou, M. Ahmad, K. Shameli, E. Saki, and K. Kalantari, Int. J. Mol. Sci. 15, 18466 (2014).

41. A.S. Perera, S. Zhang, S. Homer-Vanniasinkam, M.O. Coppens, and M. Edirisinghe, ACS Appl. Mater. Interfaces 10, 15524 (2018)

42. C. Scott, "Nylon-6 information and properties", http://www. polymerprocessing.com/polymers/PA6.html. Accessed 20 February 2018.

43. I. Goodman and A.H. Kehayoglou, Polym. J. 19, 321 (1983).

44. P. Rittigstein and J.M. Torkelson, J. Polym. Sci. Part B 44, 2935 (2006).

45. F. Rybnikář and P.H. Geil, J. Appl. Polym. Sci. 49, 1175 (1993).

46. S. Pashaei, M.M. Avval, and A.A. Syed, Chem. Ind. Chem. Eng. Q. 17, 141 (2011).

47. B. Wunderlich, Macromol. Phys. 3, 69 (2003).
48. K. Khodabakhshi, Anionic Polymarisation of Caprolactam: An Approach to Optimising the Polymerisation Condition to be Used in the Jetting Process (Doctoral dissertation, Khosrow Khodabakhshi, 2011).

49. L.P. Ramirez and K. Landfester, Macromol. Chem. Phys. 204, 22 (2003).

50. L. Ricco, S. Russo, G. Orefice, and F. Riva, Macromolecules 32,7726 (1999)

51. S. Kalia, S. Kango, A. Kumar, Y. Haldorai, B. Kumari, and R. Kumar, Colloid Polym. Sci. 292, 2025 (2014).

52. M. Doi and S.F. Edwards, The theory of polymer dynamics, vol. 73 (Oxford: Oxford University Press, 1988), pp. 140176 .

53. L.A. Feigin and D.I. Svergun, Structure analysis by smallangle $x$-ray and neutron scattering, vol. 1 (New York: Plenum, 1987), pp. 14-15.

54. J. Teixeira, J. Appl. Crystallogr. 21, 781 (1988).

55. G. Porod, in Small angle X-ray Scattering, ed. by O. Glatter, O. Kratky (London: Academic Press, 1982), p. 17.

56. K. Hino, R. Shingai, T. Morita, K. Toku, T. Hirano, H. Yoshikawa, H. Nakano, and N. Nishi, Chem. Phys. Lett. 460, 173 (2008)

57. P. Vassili, A. Evandro, N. Mario, A. Craievich, and I. Polikarpov, SAXSMoW 2.0: Online Calculator of the Molecular Weight of Proteins in Dilute Solution from Experimental SAXS Data Measured on a Relative Scale. http://sa xs.ifsc.usp.br/. Accessed 22 April 2018.

58. A. Dow, Various Applications of Magnets-Induction Coil Testing on Self-Healing Polymer Nanocomposites and ITER Tokamak. (Thesis repository, Robert Gordon University, 2018).

Publisher's Note Springer Nature remains neutral with regard to jurisdictional claims in published maps and institutional affiliations. 